\title{
Endocytoscopic findings of colorectal neuroendocrine tumors (with video) $\square$
}

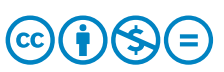

\author{
Authors \\ Kenichi Takeda', Shin-ei Kudo ${ }^{1}$, Masashi Misawa ${ }^{1}$, Yuichi Mori ${ }^{1}$, Miki Yamano ${ }^{1}$, Haruhiro Inoue ${ }^{2}$ \\ Institutions \\ 1 Digestive Disease Center, Showa University Northern \\ Yokohama Hospital, Kanagawa, Japan \\ 2 Digestive Disease Center, Showa University Koto Toyosu \\ Hospital, Tokyo, Japan \\ submitted 16.10 .2017 \\ Corresponding author \\ Shin-ei Kudo, Digestive Disease Center, Showa University \\ Northern Yokohama Hospital, Kanagawa 35-1, Chigasaki- \\ chuo Tsuzuki-ku, Yokohama-city, Kanagawa 224-8503, \\ Japan \\ Fax: +81-45-949-7535 \\ kudos@med.showa-u.ac.jp
}

accepted after revision 20.2.2018

Bibliography

DOI https://doi.org/10.1055/a-0591-9279 |

Endoscopy International Open 2018; 06: E589-E593

(c) Georg Thieme Verlag KG Stuttgart · New York

ISSN 2364-3722

\section{ABSTRACT}

Background and study aims Gastrointestinal neuroendocrine tumors (NET) are generally submucosal in location. Because these tumors are covered with normal mucosa, biopsy is necessary to confirm histological diagnosis before treatment. We explored the diagnostic capabilities of the endocytoscope, which can perform ultra-high magnification in vivo, for staining and diagnosing submucosal tumors in situ.

\section{Introduction}

Submucosal tumors (SMTs) are occasionally noted incidentally while performing colonoscopy. When an SMT is discovered, we often perform a biopsy. Gastrointestinal neuroendocrine tumors (NET) are an uncommon SMTs usually detected during endoscopic examination [1]. NETs develop from neuroendocrine cells, which are widely present in the gastrointestinal tract, and grow into the submucosa and sometimes mucosa. In the lower digestive tract, NETs are often found in the rectum during colonoscopy [2-4]. According to the histological classification of tumors developed by the World Health Organization in 2010, NETs are classified as G1 to G3 based on the rate of mitosis and the Ki-67 labeling index, and to determine the histological grade, pathological evaluation of the entire tumor is necessary [5-8]. It is necessary to distinguish NETs from other SMTs such as myoma or lipoma, which are usually not treated. It would be ideal if it were possible to diagnose SMTs from endoscopic findings to avoid excisional biopsy.

An endocytoscope is an ultra-high magnifying endoscope used to visualize living gastrointestinal cells in situ, to enable real-time endoscopic assessment of histology. Reports support the usefulness of endocytoscopy in diagnosis of epithelial neoplasia $[9,10]$. The technique has the potential to visualize tumor cells in the submucosa, because its depth of focus is $50 \mu \mathrm{m}$. We hypothesized that endocytoscopy would be useful for diagnosis of NETs because these tumors sometimes extend toward the mucosal surface layer; thus, tumor cells are likely to be observable on the surface of these lesions.

\section{Case reports}

\section{Patients and methods}

We retrospectively evaluated endocytoscopic images and pathological findings of 13 incidentally identified NETs from 13 patients evaluated endoscopically at Showa University Northern Yokohama Hospital from February 2010 to June 2014. > Table 1 shows the characteristics of the patients and lesions. UItra-high magnification images had been acquired with an integrated-type endocytoscope (CF Y-0020-I, Olympus, Tokyo, Japan) using a video endoscopic system (Evis Lucera Spectrum or Evis Lucera Elite, Olympus). To get the endocytoscopic images, $0.05 \%$ crystal violet and $1.0 \%$ methylene blue were sprayed onto the mucosa covering the lesion to stain the cytoplasm and nuclei, respectively. The lesions were resected endoscopically, with additional endocytoscopic guidance in cases with positive staining and sent for pathological diagnosis. On the basis of the pathological characteristics of NETs, cord-like 
- Table 1 Characteristics of patients and neuroendocrine tumors.

\begin{tabular}{|c|c|c|c|c|c|c|c|c|}
\hline Case & Gender & Chief complaint & Location & $\begin{array}{l}\text { Size } \\
(\mathrm{mm})\end{array}$ & $\begin{array}{l}\text { Ki-67 } \\
\text { index }\end{array}$ & $\begin{array}{l}\text { Tumor growth in } \\
\text { the intramucosa }\end{array}$ & $\begin{array}{l}\text { Distance from } \\
\text { the surface }(\mu \mathrm{m})\end{array}$ & $\begin{array}{l}\text { Tumor } \\
\text { visibility }\end{array}$ \\
\hline 1 & $\mathrm{~F}$ & Fecal occult blood & $\mathrm{Ra}$ & 5 & $<3 \%$ & - & 374.2 & - \\
\hline 2 & M & Fecal occult blood & $\mathrm{Rb}$ & 3 & $<3 \%$ & + & 10.5 & + \\
\hline 3 & M & Fecal occult blood & $\mathrm{Rb}$ & 10 & $<3 \%$ & + & 12.5 & + \\
\hline 4 & M & Constipation & $\mathrm{Rb}$ & 8 & $<3 \%$ & + & 26.3 & + \\
\hline 5 & $\mathrm{~F}$ & Screening & $\mathrm{Rb}$ & 6 & $<3 \%$ & - & 290.7 & - \\
\hline 6 & $\mathrm{~F}$ & Fecal occult blood & $\mathrm{Rb}$ & 7 & $<3 \%$ & + & 48.3 & + \\
\hline 7 & M & Constipation & $\mathrm{Rb}$ & 11 & $<3 \%$ & + & 21.6 & + \\
\hline 8 & M & Fecal occult blood & $\mathrm{Rb}$ & 6 & $<3 \%$ & + & 13.0 & + \\
\hline 9 & M & Fecal occult blood & $\mathrm{Ra}$ & 6 & $<3 \%$ & + & 18.4 & + \\
\hline 10 & $\mathrm{~F}$ & Constipation & $\mathrm{Ra}$ & 6 & $<3 \%$ & + & 36.4 & + \\
\hline 11 & M & Fecal occult blood & $\mathrm{Rb}$ & 9 & $<3 \%$ & + & 16.2 & + \\
\hline 12 & $\mathrm{~F}$ & Fecal occult blood & $\mathrm{Rb}$ & 10 & $<3 \%$ & + & 187.6 & + \\
\hline 13 & M & Constipation & $\mathrm{Rb}$ & 5 & $<3 \%$ & - & 250.6 & - \\
\hline
\end{tabular}

or honeycomb arrangements of cells with small round nuclei were defined as NETs on endocytoscopy. We assessed the diagnostic usefulness of endocytoscopy for NET by comparing endoscopic and pathological findings. The Ethics Committee of Showa University Northern Yokohama Hospital approved this study (No.17H028).

\section{Results}

All 13 tumors were in the rectum. Pathology graded all lesions as G1. In 10 lesions, tumor cells had been confirmed with endocytoscopy, but three lesions could not be confirmed using the endocytoscope and were localized based on morphology and resected using the endoscope. In the 10 endocytoscopicallyconfirmed lesions, cells with compact, homogeneous, circular nuclei were arranged in a cord-like or honeycomb array around the epithelial ducts. The cytoplasm did not stain with crystal violet. Vessels running between the tumor cell arrays were observed on a video image generated by the endocytoscopic system ( $\vee$ Video 1$)$. Endoscopic findings were consistent with characteristic pathological signs of NET. Pathology confirmed the findings ( $\triangleright$ Fig. 1 ).

We measured the distance from mucosa to the tumor cells on the pathological sections ( $>$ Table 1 ). Of 10 lesions in which the tumor could be observed with endocytoscopy, there was tumor within $50 \mu \mathrm{m}$ of the mucosal surface in nine lesions. In one lesion, tumor deeper than $50 \mu \mathrm{m}$ was observed. In the three lesions in which tumor cells were not observed with endocytoscopy, the depth from the mucosa to the tumor cells was $>50 \mu \mathrm{m}$.

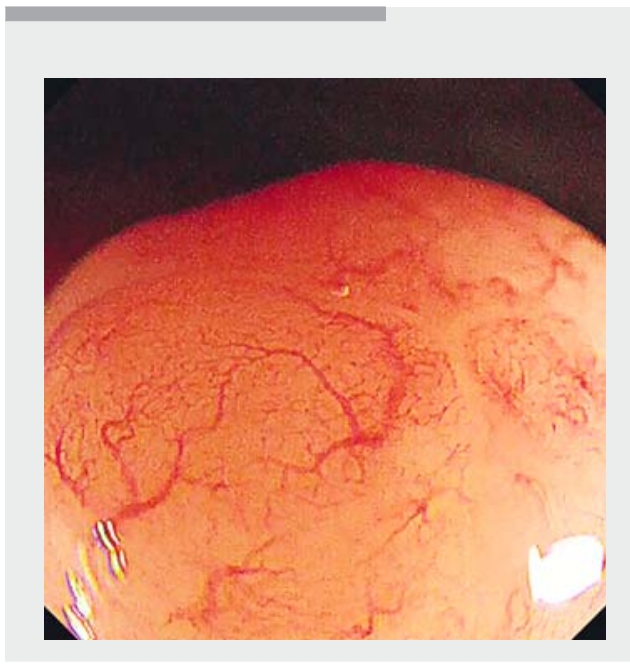

Video 1 An endocytoscopic video observing neuroendocrine tumor. The lesion was a submucosal tumor exhibiting a yellowish-white color, $6 \mathrm{~mm}$ in diameter. Blood flow is apparent in the vessels stretched over the surface layer. In endoscopic observation, mucosal defects are not observed in the lesion. After staining the lesion with crystal violet and methylene blue, ultra-high magnifying endoscopy commences. Cells with small, round stained nuclei are arranged in a cord-like array, and blood flow is observed in vessels surrounded by the cord-like structures.

\section{Discussion}

In the case of SMTs covered with normal mucosa, the operator can only infer the pathological diagnosis from the color and hardness of the tumor [5]. In this study, we were able to observe cord-like or honeycomb arrays of cells in 10 of 13 lesions using endocytoscopy, and these findings corresponded with 

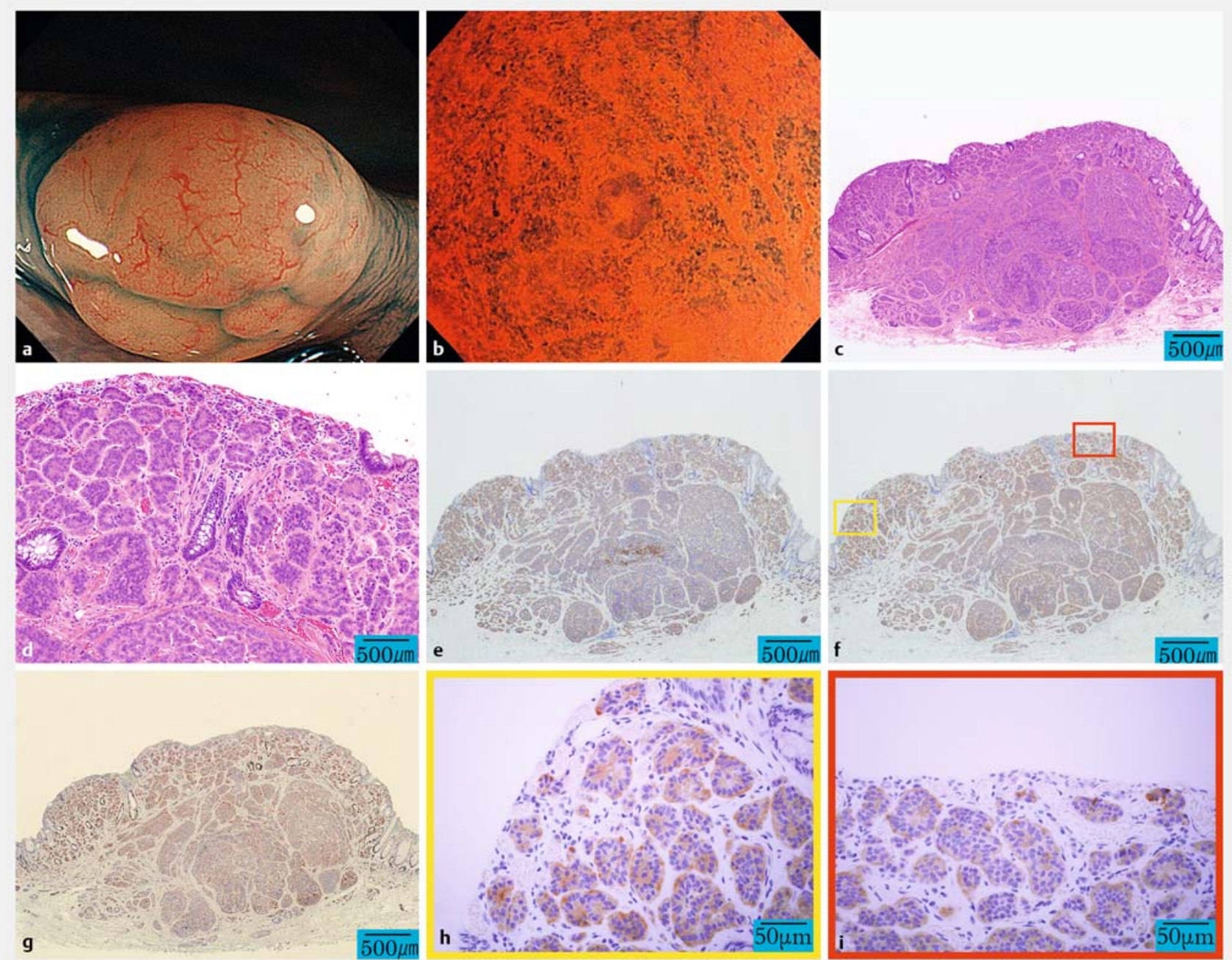

- Fig.1 Endocytoscopic and pathological findings in Case 8. a Indigo carmine-sprayed endocytoscopic image. b Endocytoscopic image stained with $0.05 \%$ crystal violet (CV) and $1.0 \%$ methylene blue (MB). c and d Histologic sections stained with hematoxylin \& eosin (HE). Tumor cells are arranged in honeycomb- and cord-like patterns around the submucosa. e Chromogranin A-positive cells are present. f Synaptophysin-

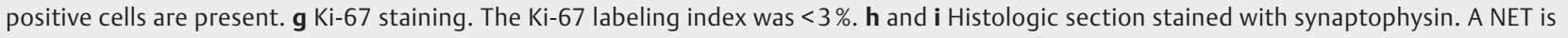
located near the mucosal surface but is not exposed.

findings on pathology. As NETs enlarge in the submucosa, they extend beneath the superficial mucosa, stretching it and resulting in the crypts becoming sparser and thinning of the surface mucous membrane. We postulated that stretching and thinning of the interstitial epithelium between the crypts would bring these tumors into the depth of focus of the endocytoscope, allowing examination. However, such extension of the tumor to beneath the surface layer was not detected by endocytoscopy or pathological examination of specimens in our study cohort ( $\vee$ Video $\mathbf{1}$, $\mathbf{F i g .} \mathbf{1}$ ).

Nine out of 10 diagnosed cases featured NET cells within $50 \mu \mathrm{m}$ of the tumor surface, which can be explained by the fact that the focal depth design of the endocytoscope is $50 \mu \mathrm{m}$. Among the lesions identified by endocytoscopy, there was one lesion in which tumor cells were $>50 \mu \mathrm{m}$ from the surface layer $(\triangleright$ Table 1, Case 12). In this tumor, the epithelial duct morphology was distorted. Compression of the lesion with the lens stretched the mucous membrane more thinly, enabling visualization of this tumor. In addition, similarly to the lesions that could be observed with EC, in Case 12, the tumor had extended into the superficial mucosal layer.

Endocytoscopy could not identify tumors in three NETs located much deeper than $50 \mu \mathrm{m}$ from the surface layer, indicating that not all NETs can be detected with endocytoscopy ( $\triangleright$ Fig. 2 ). In addition, we did not observe tumor growth in the surface layer of the mucosa. It was not possible to examine tumors deep to the epithelial ducts, suggesting that presence of tumors in the surface layer influences their visibility.

This study has some limitations. It was retrospective study and from a single center. We evaluated only NET G1 SMTs; other submucosal tumors were not included because we had no endocytoscopic images of G2, G3, or other submucosal tumors. Because the cytoplasm did not stain with crystal violet, we could visualize only the nuclei, which stained with methylene 

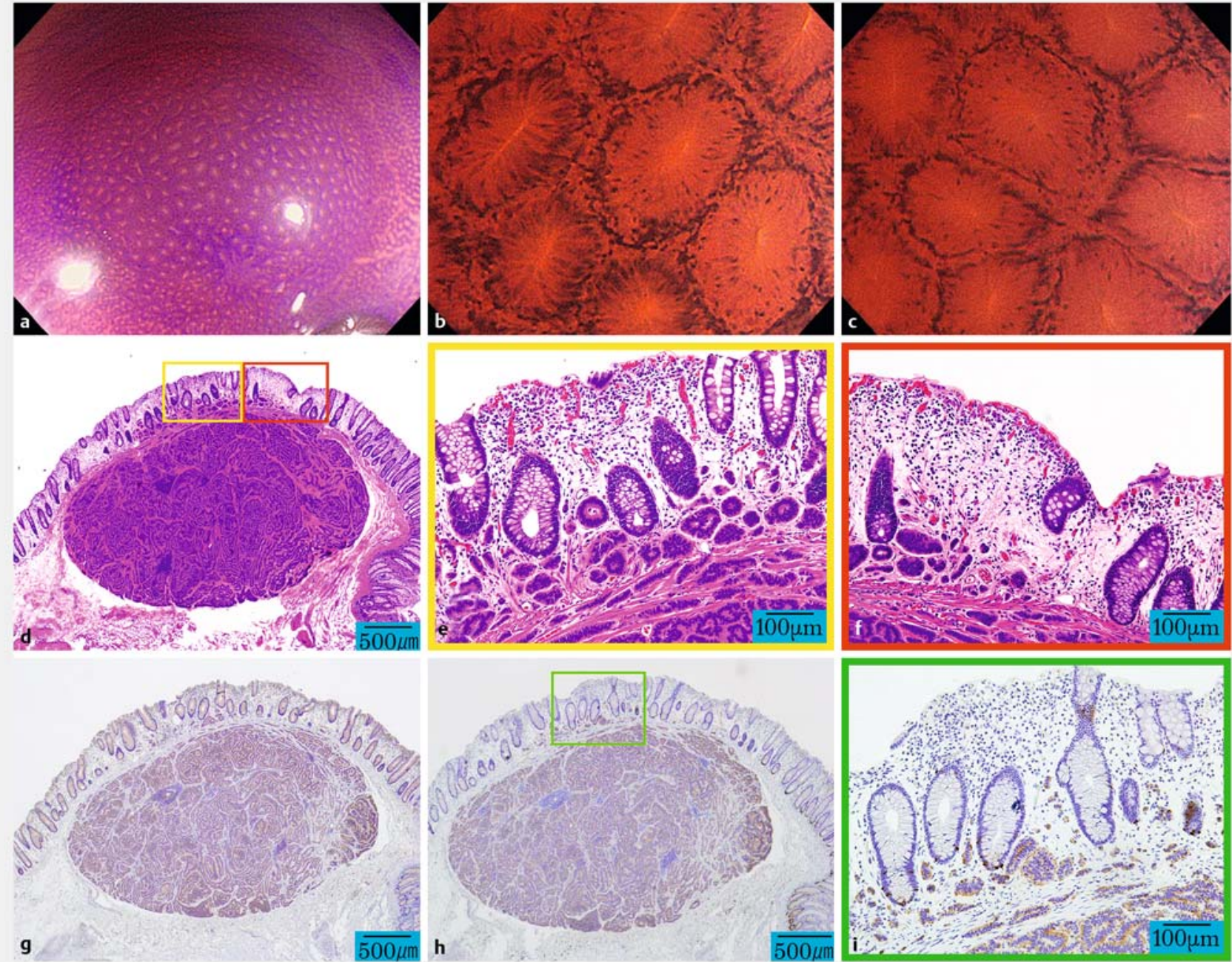

- Fig. 2 Endoscopic and pathological findings in Case 5. a An endocytoscopic image of a lesion stained with CV. b and c Endocytoscopic images. Dense mucosal epithelial cells are apparent. $\mathbf{d}$, e, and $\mathbf{f}$ Histologic sections stained with HE. A NET is situated mainly in the submucosal layer, most of its cells being deep to the crypts. $\mathbf{g}$ Synaptophysin-positive cells are visible. $\mathbf{h}$ and $\mathbf{i}$ Chromogranin-A positive cells are visible in the deep mucosal and submucosal layers.

blue. The small abnormal nuclei were arranged in a cord-like or honeycomb array, a finding characteristic of NET that was supported by pathological findings. In a biopsy, a section perpendicular to the mucosal surface image can be evaluated by pathological examination, whereas endocytoscopy mainly enables observation of tumors in a plane parallel to the mucosal surface, making it impossible to fully correlate endocytoscopic images and pathological findings.

\section{Conclusion}

The possibility that endocytoscopy can be used for examination of NETs suggests a new indication for this methodology. Examination of more lesions in the future may enable diagnosis of them in situ and assessment of the need for treatment without taking invasive biopsies.

\section{Acknowledgements}

We thank Olympus (Tokyo, Japan) for their cooperation in providing information on endocytoscopy. We thank Libby Cone, MD, MA, from Edanz Group Japan (www.edanzediting.com/ac) for editing a draft of this manuscript.

Competing interests

None

References

[1] Shim KM, Yang SK, Myung SJ et al. Atypical endoscopic features of rectal carcinoids. Endoscopy 2004; 36: 313 -316

[2] Jernman J, Välimäki MJ, Louhimo J et al. The novel WHO 2010 classification for gastrointestinal neuroendocrine tumours correlates well 
with the metastatic potential of rectal neuroendocrine tumours. Neuroendocrinology 2012; 95: 317 - 324

[3] Ito T, Sasano H, Tanaka M et al. Epidemiological study of gastroenteropancreatic neuroendocrine tumors in Japan. J Gastroenterol 2010; 45: $234-243$

[4] Gleeson FC, Levy MJ, Dozois EJ et al. Endoscopically identified welldifferentiated rectal carcinoid tumors: impact of tumor size on the natural history and outcomes. Gastrointest Endosc 2014; 80: 144 151

[5] Matsui K, Iwase T, Kitagawa M. Small, polypoid-appearing carcinoid tumors of the rectum: clinicopathologic study of 16 cases and effectiveness of endoscopic treatment. Am J Gastroenterol 1993; 88: $1949-1953$

[6] Ono A, Fujii T, Saito Y et al. Endoscopic submucosal resection of rectal carcinoid tumors with a ligation device. Gastrointest Endosc 2003; 57: $583-587$
[7] Lee DS, Jeon SW, Park SY et al. The feasibility of endoscopic submucosal dissection for rectal carcinoid tumors: comparison with endoscopic mucosal resection. Endoscopy 2010; 42: 647 - 651

[8] Rindi G, Arnold R, Bosman FT et al. Nomenclature and classification of neuroendocrine neoplasms of the digestive system. In: Bosman FT, Carneiro F, Hruban R, Theise ND eds. WHO classification of tumours of the digestive system. Geneva: World Health Organization; 2010

[9] Kudo SE, Wakamura K, Ikehara N et al. Diagnosis of colorectal lesions with a novel endocytoscopic classification - a pilot study. Endoscopy 2011; 43: $869-875$

[10] Takeda K, Kudo SE, Mori Y et al. Magnifying chromoendoscopic and endocytoscopic findings of juvenile polyps in the colon and rectum. Oncol Lett 2016; 11: $237-242$ 